\title{
Recovery from paraplegia with administration of erlotinib in a patient with lung adenocarcinoma
}

\author{
Koichi Kurishima ${ }^{1}$, Katsunori Kagohashi ${ }^{1}$, Takeo Mammoto ${ }^{2}$, Hiroaki Satoh ${ }^{1}$ \\ 'Division of Respiratory Medicine, Mito Medical Center, University of Tsukuba, Japan \\ ${ }^{2}$ Division of Orthopedics, Mito Medical Center, University of Tsukuba, Japan
}

Contemp Oncol (Pozn) 2014; 18 (2): 140-142 DOI: $10.5114 /$ wo.2014.41393

Paraplegia caused by spinal cord compression (SCC) due to vertebral metastasis is considered an oncological emergency, and it requires immediate treatment [1-5]. Irradiation of the metastatic lesion and corticosteroid therapy are usually carried out, but the possibility of controlling the lesion is very low [3]. Erlotinib is a molecular targeted drug widely used to treat patients with advanced non-small cell lung cancer (NSCLC), and its clinical utility is highly evaluated [4]. We herein report a paraplegic patient successfully treated with erlotinib in addition to such palliative therapies.

A 77-year-old man was admitted to our hospital with gradual onset of weakness and dysesthesia of the bilateral lower extremities, bladder and bowel disturbance, and back pain of 1-month duration. He was a never smoker. Nine years previously, he had been diagnosed as having adenocarcinoma in the lower lobe of the right lung. As there was no distant metastasis, he received lobectomy and mediastinal lymph node dissection. The pathological staging was T1aNOMO, stage IA (seventh edition of the TNM classification), lepidic-predominant adenocarcinoma. But EGFR mutation was not analyzed at that time. Two months after the surgery, follow-up chest CT scan revealed left pretracheal lymph node recurrence. He received radiotherapy and platinum-containing chemotherapy. At the follow-up 3 years after this additional therapy, he had again developed local recurrence in the left lung. This time, he was treated with gefitinib $(250 \mathrm{mg} / \mathrm{d})$. One year after the initiation of gefitinib, he noticed numbness in the area of both knees, which was rapidly progressing to paraplegia. On admission to our hospital, he was unable to walk. Bladder and bowel disturbance was also observed. Additionally, he complained of dysesthesia of the anterior aspects of both legs. Neurologic examination revealed bilateral weakness of the hip adductors, quadriceps femoris, adductor muscles, and posterior tibial muscle (muscle strength grade I/V on the right, II/V on the left). There were no signs of upper neuron damage. Lower extremity reflexes were reduced bilaterally. No pathologic reflexes were noted. Magnetic resonance imaging (MRI) showed vertebral metastases at Th9 and S1, which had destroyed the bodies of these vertebrae, causing SCC (Fig. 1). A metastatic workup to find involvement of the other organs was negative. The lesions were managed with dexamethasone and palliative radiotherapy of up to 36 Gy, but the patient's lower limb muscle weakness did not improve. Therefore, we replaced the gefitinib with erlotinib (150 $\mathrm{mg} / \mathrm{d})$. At the follow-up 2 months after initiation of erlotinib, the neurologic status showed distinct improvement insofar as the patient was able to walk a short distance on his own. Improvement of the bladder and bowel disturbance was also observed. The follow-up MRI 2 months after the initiation of erlotinib showed a significant decrease in the size of the vertebral metastases and improvement of the SCC (Fig. 2). He was regularly monitored in our pulmonary division for 4 months. However, his health deteriorated. He was admitted to our hospital again and died of lung cancer 1 month later.

Metastatic SCC is defined as "spinal cord or cauda equina compression by direct pressure, and/or induction of vertebral collapse or instability by metastatic spread or by direct extension of malignancy that threatens or causes neurological disability" [1]. Metastatic SCC, a complication of skeletal metastasis, is a medical emergency with potentially debilitating and disastrous outcomes [1-5]. Because improved diagnostic and therapeutic tools have prolonged patient survival, vertebral metastasis and metastatic SCC have become important clinical, ethical, epidemiological and economic concerns. More than $5 \%$ of cancer patients will develop metastatic SCC during their disease course, the incidence varying considerably with the primary tumor histology $[2,6]$. The common primary malignancies causing metastatic SCC are myeloma and breast, lung, prostate and kidney cancers $[2,6]$. The prognosis of patients with metastatic SCC depends on the primary tumor, and survival for those with tumors such as lung cancer, melanoma and sarcoma is generally dismal [7]. Treatment options for metastatic SCC include surgery and radiotherapy. If the patient proves to have metastatic SCC, he/she requires immediate treatment. As most patients with metastatic SCC are not suitable for surgery, irradiation of the metastatic site of the vertebra is the most commonly used option [2, 8]. Many patients with metastatic SCC due to vertebral metastasis initially receive irradiation of the metastatic lesion and corticosteroid therapy, but rarely with remarkable effect [3]. In a recent review by Mehta [9], clinical evidence for the effectiveness of whole brain radiation therapy (WBRT) with concurrent erlotinib [10] and of WBRT with sequential erlotinib [11] was presented. However, neither radiation combined with erlotinib for metastatic spinal tumors nor that for metastatic vertebral tumors has been reported. In our patient, irradiation of the metastatic site and dexamethasone were selected because of his poor 


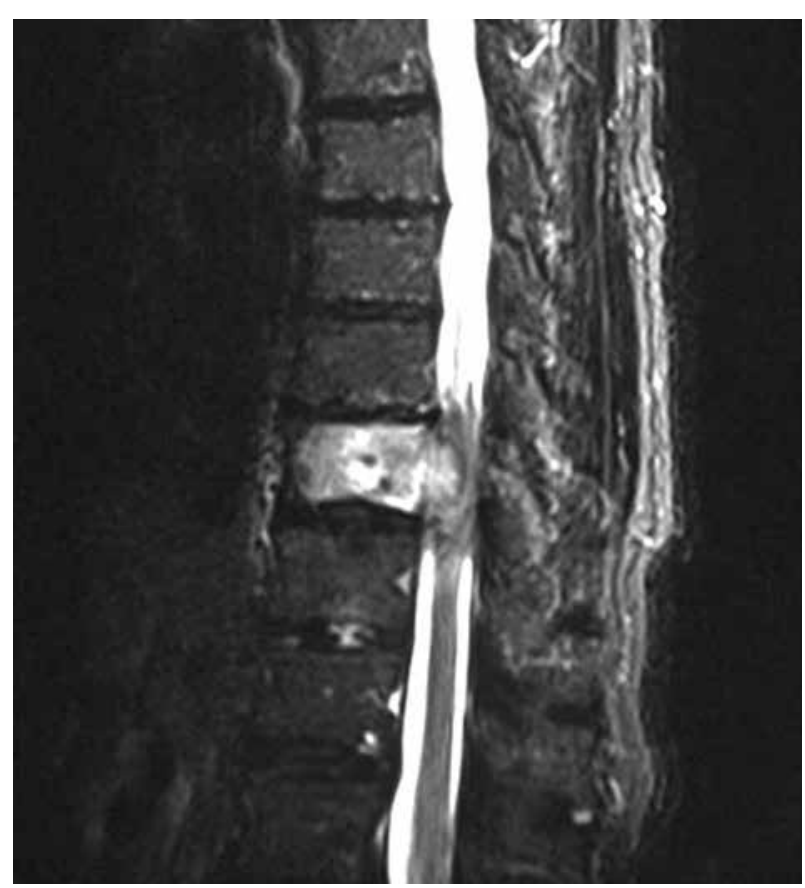

Fig. 1. Spinal sagittal T2-weighted MR image at admission showing spinal cord compression by a vertebral metastasis at the Th9 level

performance status, but his lower limb muscle weakness was not improved with these treatments. Two months after the initiation of erlotinib, however, the neurologic status showed distinct improvement insofar as he was able to walk a short distance on his own. Improvement of the bladder and bowel disturbance was also observed.

Gefitinib and erlotinib are 2 small-molecule epidermal growth factor receptor tyrosine kinase inhibitors (EGFR-TKIs) that have been approved for the treatment of NSCLC [4]. Gefitinib and erlotinib have similar mechanisms of action and pharmacologic profiles; however, their different molecular structures confer pharmacokinetic differences that may have important clinical implications. Gefitinib or erlotinib EGFR-TKIs are active only in EGFR-mutant lung cancers. Therefore, evaluation of the EGFR gene is currently mandatory in the treatment of patients with advanced NSCLC. However, our patient was not evaluated for EGFR mutation because the evaluation was not common at the time of his surgical resection.

Some researchers have reported the efficiency of erlotinib as second- or later-line treatment after cytotoxic chemotherapy $[12,13]$ or after gefitinib therapy [15-17]. According to a review by Saito et al., the response rate ranged from $4.3 \%$ to $25 \%$, and the disease control rate ranged from $8.7 \%$ to $75 \%$ [16]. Koma et al. reported a patient successfully treated with erlotinib after gefitinib-induced interstitial lung disease [17]. We speculated that the efficiency of erlotinib after gefitinib might be due to the fact that the accepted dosages of gefitinib $(250 \mathrm{mg} / \mathrm{d})$ and erlotinib $(150 \mathrm{mg} / \mathrm{d})$ are not equivalent [18] and that such a dose of gefitinib produced lower mean plasma concentrations and area under the plasma concentration versus time curves than did the daily dose of erlotinib [19]. In addition, gefitinib was administered at only one-third to one-sixth of its maximum dosage; on the other hand, erlotinib was administered at its maximum dosage [20].

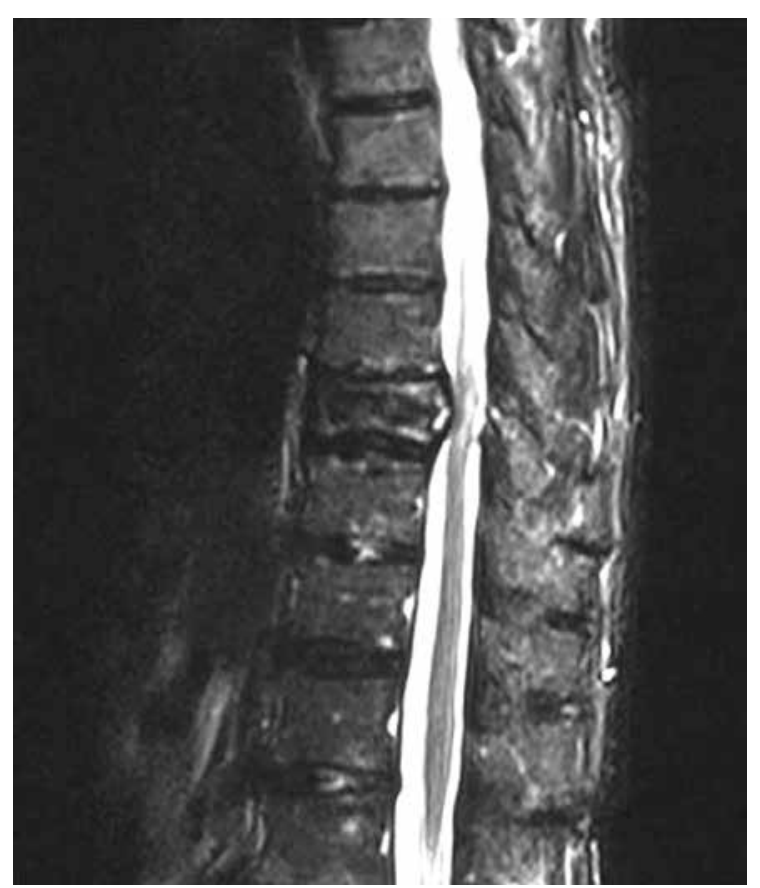

Fig. 2. The 2-month follow-up T2-weighted MR image after the initiation of erlotinib showing a significant decrease in the size of the vertebral metastasis and improvement of the spinal cord compression

Very recently, second-generation irreversible EGFR-TKIs such as afatinib (BIBW2992) have attracted attention. Ou summarized the theoretical advantages of second-generation irreversible EGFR-TKIs over first-generation reversible EGFR TKIs as being that some have a higher affinity for the EGFR kinase domain and an irreversible tyrosine kinase blockade may result in longer suppression of ErbB signaling than that resulting from reversible inhibitors [21]. In addition, second-generation EGFR TKIs inhibit human epidermal growth factor receptor 2 (HER2), a common dimerization partner of EGFR, and some inhibit HER4 as well, to affect signaling transduction, thus allowing a more complete blockade of the EGFR signaling pathway [21]. Second-generation EGFR TKIs also have modest in vitro activity against the $T 790 \mathrm{M}$ gatekeeper mutation and other rare mutations that render first-generation reversible EGFR TKIs ineffective [21]. These properties might be related to the mechanism of action of second-generation irreversible EGFR-TKIs after first-generation TKIs.

As for bone metastasis from NSCLC, gefitinib inhibited tumor cell proliferation at bone metastatic sites and induced normal bone formation in some previous reports [22, 23]. To the best of our knowledge, however, the effectiveness of erlotinib against metastatic SCC due to vertebral metastasis, which was uncontrolled by gefitinib, has not been reported. We therefore determined that this is the first case in which significant improvement of paraplegia could be achieved with erlotinib treatment. Here we have reported a rare case of paraplegia successfully treated with erlotinib in addition to radiotherapy. Although this is only one case, erlotinib might be considered as a treatment option when no other promising therapy is available.

The authors declare no conflict of interest. 


\section{References}

1. Prewett S, Venkitaraman R. Metastatic spinal cord compression: review of the evidence for a radiotherapy dose fractionation schedule. Clin Oncol (R Coll Radiol) 2010; 22: 222-30.

2. Loblaw DA, Mitera G, Ford M, Laperriere NJ. A 2011 updated systematic review and clinical practice guideline for the management of malignant extradural spinal cord compression. Int J Radiation Oncol Biol Phys 2012; 84: 312-7.

3. Ruckdeschel JC. Early detection and treatment of spinal cord compression. Oncology (Williston Park) 2005; 19: 81-6.

4. Govindan R. A review of epidermal growth factor receptor/HER2 inhibitors in the treatment of patients with non-small-cell lung cancer. Clin Lung Cancer 2010; 11: 8-12.

5. Guideline NICE. Metastatic spinal cord compression: diagnosis and management of patients at risk of or with metastatic spinal cord compression. UK: National Institute of Health and Clinical Excellence, $\mathrm{Na}$ tional Collaborating Centre for Cancer; 2008.

6. Zaikova O, Giercksky KE, FossåSD, Kvaløy S, Johannesen TB, Skjeldal S. A population-based study of spinal metastatic disease in South-East Norway. Clin Oncol (R Coll Radiol) 2009; 21: 753-9.

7. Rades D, Fehlauer F, Schulte R, et al. Prognostic factors for local control and survival after radiotherapy of metastatic spinal cord compression. J Clin Oncol 2006; 24: 3388-93.

8. Levack P. A prospective audit of the diagnosis, management and outcome of malignant cord compression. 97/08. London: CRAG, NICE Clinical Audit \& Resource Group; 2001.

9. Mehta VK. Radiotherapy and erlotinib combined: review of the preclinical and clinical evidence. Front Oncol 2012; 2: 31. doi: 10.3389/ fonc.2012.00031

10. Lind JS, Lagerwaard FJ, Smit EF, Senan S. Phase I study of concurrent whole brain radiotherapy and erlotinib for multiple brain metastases from non-small-cell lung cancer. Int J Radiat Oncol Biol Phys 2009; 74: 1391-6.

11. von Pawel J, Wagner H, Duell T, Poellinger B. Erlotinib in patients with previously irradiated, recurrent brain metastases from non-small cell lung cancer: two case reports. Onkologie 2008; 31: 123-6.

12. Triano LR, Deshpande H, Gettinger SN. Management of patients with advanced non-small cell lung cancer: current and emerging options. Drugs 2010; 70: 167-79.

13. Kowalski DM, Krzakowski M, Ramlau R, Jaskiewicz P, Janowicz-Żebrowska A. Erlotinib in salvage treatment of patients with advanced non-small cell lung cancer: results of an expanded access programme in Poland. Wspolczesna Onkol 2012; 16: 170-5.

14. Garfield DH. Modern treatment of lung cancer: case 2. Response to erlotinib after failure of gefitinib in a patient with advanced non-small-cell lung carcinoma. J Clin Oncol 2005; 23: 7778-840.

15. Walther JC, Khorshid M, Gaya A, Plowman PN. Cross-over response to erlotinib of brain metastatic disease from bronchial adenocarcinoma after gefitinib failure, and an unusual rash. Clin Oncol (R Coll Radiol) 2006; 18: 637-9.

16. Saito H, Murakami S, Kondo T, Oshita F, Noda K, Yamada K. Effectiveness of erlotinib in advanced non-small cell lung cancer in cases of gefitinib resistance after treatment of more than 6 months. Onkologie 2012; 35: 18-22.

17. Koma Y, Matsuoka H, Yoshimatsu H, Suzuki Y. Successful treatment with erlotinib after gefitinib-induced interstitial lung disease: a case report and literature review. Int J Clin Pharmacol Ther 2012; 50: 760-4.

18. Paz-Ares L, Souli res D, Melezínek I, Moecks J, Keil L, Mok T, Rosell R, Klughammer B. Clinical outcomes in non-small-cell lung cancer patients with EGFR mutations: pooled analysis. J Cell Mol Med 2010; 14: 51-69.

19. Rukazenkov Y, Speake G, Marshall G, Anderton J, Davies BR, Wilkinson RW, Mark Hickinson D, Swaisland A. Epidermal growth factor receptor tyrosine kinase inhibitors: similar but different? Anticancer Drugs 2009; 20: 856-66.

20. Yeo WL, Riely GJ, Yeap BY, et al. Erlotinib at a dose of $25 \mathrm{mg}$ daily for non-small cell lung cancers with EGFR mutations. J Thorac Oncol 2010; 5: 1048-53.

21. Ou SH. Second-generation irreversible epidermal growth factor receptor (EGFR) tyrosine kinase inhibitors (TKIs): a better mousetrap? A review of the clinical evidence. Crit Rev Oncol Hematol 2012; 83: 407-21.
22. Zukawa M, Nakano M, Hirano N, Mizuhashi K, Kanamori M. The effectiveness of gefitinib on spinal metastases of lung cancer: report of two cases. Asian Spine J 2008; 2: 109-13.

23. Satoh H, Ishikawa H, Ohara G, Kikuchi N, Sekizawa K. Prolonged response to gefitinib in bone metastasis. Med Oncol 2009; 26: 101-2.

\section{Address for correspondence}

\section{Hiroaki Satoh MD}

Division of Internal Medicine

Mito Medical Center, University of Tsukuba

Miya-machi 3-2-7, Mito

Ibaraki, 310-0015, Japan

tel. +81292312371

fax +81292215137

e-mail: hirosato@md.tsukuba.ac.jp

Submitted: 20.03 .2013

Accepted: 16.07 .2013 